\title{
Determination of surface defects by using the wavefront scanner
}

\author{
N.S. Goloborodko ${ }^{1}$, V.I. Grygoruk, V.N. Kurashov, D.V. Podanchuk, A.A. Goloborodko ${ }^{2}$, M.M. Kotov ${ }^{3}$ \\ Taras Shevchenko Kyiv National University, Department of Radiophysics, Optical Processing Laboratory, \\ 64, Volodymyrska str., 01033 Kyiv, Ukraine \\ E-mail:sunata@univ.kiev.ual,angol@univ.kiev.ua ${ }^{2}$,mkot@univ.kiev.ua ${ }^{3}$
}

\begin{abstract}
The possibility of changes in the polarization state of the laser beam reflected from inhomogeneity with the refractive index gradient is theoretically shown, which allows separating the phase shifts related with relief inhomogeneities and local changes of the surface refractive index. Modification of the wavefront scanner for analyzing the wavefront of the laser beam reflected from the samples' surface is considered. The main idea of the method is to use the focused laser beams with different polarizations for illuminating separate areas of the surface. The results of detecting test surfaces with different structures by the wavefront scanner are presented.
\end{abstract}

Keywords: wavefront scanner, surface defects, polarization.

Manuscript received 19.10.09; accepted for publication 22.10.09; published online 30.12.09.

\section{Introduction}

In recent years, the problem of contactless diagnostics of surfaces became extremely urgent. Optical methods of investigating the structured objects provide measuring and analyzing the wavefront of the laser beam reflected from their surface. Among the wavefront sensors the Shack-Hartmann sensor should be mentioned as the most used [1,2]. The principle of its work is well known [3]. The main obstacle in application of these sensors for precision monitoring of the surface is their insufficient spatial resolution that is determined by the lenslet size. One of the methods for solving this problem is the use of a focused laser beam that after reflection from the tested surface passes through the same optical scheme and gets to the sensor [4]. In this case, the signal formed in the sensor plane can be treated as the phase image of the surface subarea, and the spatial resolution is determined only by the sensor aperture [5]. Though in the case of measuring with this scheme the tested wavefront is almost plane, that requires a high spatial sensitivity of the device that cannot be always provided by the sensor. The alternative to this can be the wavefront scanner $[6,7]$ that differs from the sensor in the fact that the lenslet array is replaced by one lens with longer focal distance and with controlled entrance aperture. Owing to this, the angular sensitivity of the scanner can be sufficiently enlarged.

It should be mentioned that in wavefront measuring the phase distribution in the observation plane is supposed to be related with changing the tested surface shapes. As a rule, it is supposed in this case that the phase distribution in the observation plane is directly concerned with the tested sample shape variations [8]. Besides in microelectronics, where the tested surface has the complicated semiconductor structure, the phase distribution of the reflected wave depends not only on geometric phase shift, which is caused by local inhomogeneities on the sample, but also on electronic structure of separate surface elements [9]. In the ordinary registration scheme, these components cannot be separated, which makes it impossible not only the confident surface shape identification but also identifying the influence of material factors on the phase distribution of the field reflected from the structured surface.

The Hartmann wavefront scanner modification that allows overcoming this ambiguity, i.e. to separate phase shifts, which are caused by surface shape geometry and sample material structure is considered in this work. The main idea of the method is to use focused laser beams with different polarizations for illuminating separate areas of the surface. Then the difference in wavefront shape that is registered during the illumination with different polarization allows separating the corresponding phase components caused by the factors mentioned above.

\section{Theory and equations}

The main feature of the proposed experimental method is the use of focused laser beam which after reflecting 
from the sample reflects back, passes again the focusing system and forms the signal in the sensors plane that is the phase image of the illuminated spot on the surface (Fig. 1a). Let's consider the process of formatting the secondary optical field that is reflected from the inhomogeneity (Fig. 1b).

The specificity of this method is that information about relief of the reflecting surface is transposed to the phase information of the scattered component. The mathematical model of optical system mentioned above is determined by the following considerations:

1. Input wavefront is supposed to be plane (this assumption corresponds to usual practice of metrological investigations, i.e. observation of infinitely far source of light).

2. Forming system is supposed to be ideal, i.e., that does not insert additional aberrations into the tested wavefront.

We will describe the model of optical system by the linear transform of input wavefront $U_{0}(\vec{r})$ :

$U_{\mathrm{I}}(\vec{\rho})=\int U_{0}(\vec{r}) h_{\mathrm{I}}(\vec{r}, \vec{\rho}) d^{2} \vec{r}$,

where $U_{\mathrm{I}}(\vec{\rho})$ is the distribution of field complex amplitude in the probing plane and the point-spread function is given by:

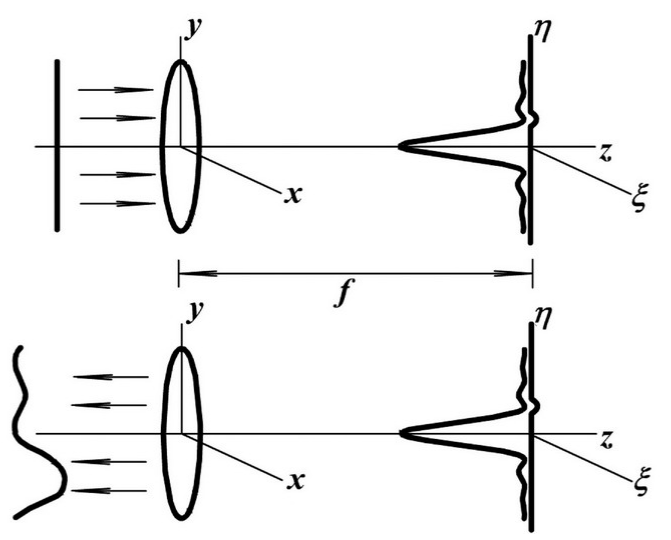

a)

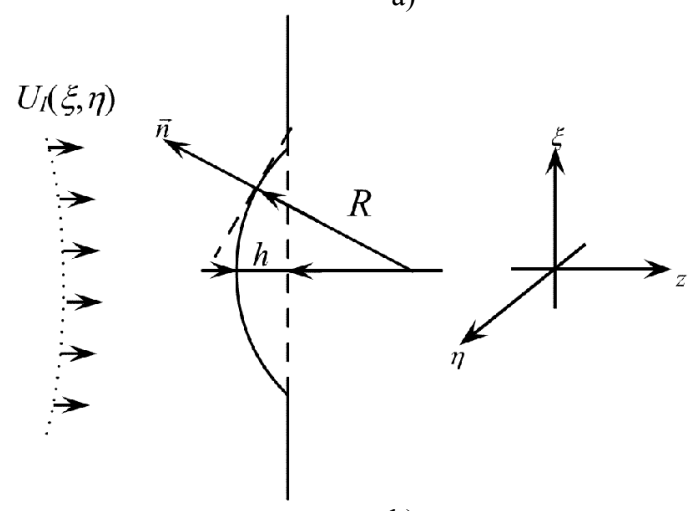

b)

Fig. 1. The process of the secondary field formation: (a) double-pass system and (b) scheme of formation of the additional phase incursion from the surface defect. $h(\vec{r}, \vec{\rho})=\frac{1}{j \lambda r} e^{j \frac{2 \pi}{\lambda}|\vec{r}-\vec{\rho}|} \cos (\vec{k}, \vec{r}-\vec{\rho})$,

and is the pulse reaction of ideal focusing system. Here, $\lambda$ is the wavelength of the probing beam, $r$ describes the coordinates in the forming plane, $\rho$ describes coordinates in the scanning plane, $\vec{k}$ is the direction of wave vector of the incident wave. Distribution of the complex amplitude in the scanning plane can be analytically written as a Fourier transform of the optical signal with the constant phase [10]. It should be mentioned that in the case of reflecting from the plane surface, the outlet wave will be flat, since the inverse transform is equivalent to the direct one, but if the testing surface has some inhomogeneity, then the reflected wave includes a multiplier that corresponds to reflection from this inhomogeneity. On the other hand, it should be mentioned that accounting of only phase incursions do not allow precise description of the experimental results where the amplitude distribution change is observed together with the wave phase structure changes. To determine the influence of phase incursions from the inhomogeneity, taking into consideration the direction of reflecting and reflectance, let us consider the case of reflection from the spherical inhomogeneity with characteristic sizes $R$ and $h$ (Fig. 1b), which inserts an additional phase disturbance into the reflected wave, and the reflection from the mentioned defect can be written as:

$\mathfrak{R}(\xi, \eta)=M(\xi, \eta) \mathrm{e}^{j \frac{2 \pi}{\lambda R}\left(\xi^{2}+\eta^{2}\right)}$,

where $M(\xi, \eta)$ is the coefficient of reflection from the surface, and, in general case, this coefficient depends on the reflectance of the material and the angle between the wave vector of the incident wave and normal to the chosen point on the surface. The array of nonzero reflecting coefficients $M$ can be written as:

$$
\begin{aligned}
& M_{\xi \xi}=R_{\perp} \frac{\left(1-Z_{\xi}^{\prime 2}\right) k_{i n \xi}-2 k_{i n z} Z_{\xi}^{\prime}}{1+Z_{\eta}^{\prime 2}+Z_{\xi}^{\prime 2}} \mathrm{e}^{j \frac{2 \pi}{\lambda R}\left(\xi^{2}+\eta^{2}\right)}, \\
& M_{z \xi}=R_{\perp} \frac{\left(1-Z_{\xi}^{\prime 2}\right) k_{i n z}+2 k_{i n \xi} Z_{\xi}^{\prime}}{1+Z_{\eta}^{\prime 2}+Z_{\xi}^{\prime 2}} \mathrm{e}^{j \frac{2 \pi}{\lambda R}\left(\xi^{2}+\eta^{2}\right)}, \\
& M_{\eta \eta}=R_{\|} \frac{\left(1-Z_{\eta}^{\prime 2}\right) k_{i n \eta}-2 k_{i n z} Z_{\eta}^{\prime}}{1+Z_{\eta}^{\prime 2}+Z_{\xi}^{\prime 2}} \mathrm{e}^{j \frac{2 \pi}{\lambda R}\left(\xi^{2}+\eta^{2}\right)}, \\
& M_{z \eta}=R_{\|} \frac{\left(1-Z_{\eta}^{\prime 2}\right) k_{i n z}+2 k_{i n \eta} Z_{\eta}^{\prime}}{1+Z_{\eta}^{\prime 2}+Z_{\xi}^{\prime 2}} \mathrm{e}^{j \frac{2 \pi}{\lambda R}\left(\xi^{2}+\eta^{2}\right)},
\end{aligned}
$$

where $R_{\perp}$ and $R_{\|}$are Fresnel local reflection coefficients. It should be mentioned that the reflection 
coefficient will be influenced by the refraction index and absorption factor, optical path length, phase relations between them that define the interference conditions. Sure, since the surface inhomogeneities can be the local variations of the refraction index, then taking them into account leads not only to the amplitude changes, but also causes rotation of the polarization axis. As an example, it is enough to consider the optical beam pass in the stratified structure. Taking into account the interference after passing each boundary (Fig. 2), absorption in each layer and also the averaging within the aperture, basing on the electromagnetic theory of light we deduced equations shown below for the complex amplitudes of the reflected and refracted waves in the terms of optical macroscopic characteristics for these structures.

In the case of using optical radiation, one can move from the dielectric and magnetic permittivity to the description of light interaction with such a structure by using the complex refractive index $n=n_{\mathrm{Re}}+j n_{\mathrm{Im}}$, where $n_{\mathrm{Re}}, n_{\mathrm{Im}}$ are refractive and absorption indices, correspondingly. Therefore, if the Fresnel coefficients of the reflection and transmission at the boundary between media with different reflective indexes are known, then the normalized complex reflection coefficient could be calculated as:

$R=r^{0,1}+\sum_{i=1}^{N} \prod_{k=1}^{i}\left(t^{k-1, k} e^{j \frac{2 \pi d_{k}}{\lambda \cos \varphi_{k}}}\right)^{2} r^{i-1, i}$,

where $r^{i-1, i}$ and $t^{i-1, i}$ are complex reflection and transmittance coefficients on the boundary between $i-1$ and $i$ layers, $d_{i}$ is the depth of $i$ layer, $\varphi_{t_{i}}$ is the angle of incidence on the boundary $i-1, i, N$ is the number of layers. As one can see, the normalized reflection coefficient includes the phase multiplier that corresponds to the phase incursion of the wave when spreading in the multilayer medium. This proofs the ability of the interference appearance and is revealed as oscillations of reflection coefficient angular dependence. Examples of such oscillations are shown in Fig. 3a.

Thus, it is obvious that each set of angular dependences is uniquely defined by the multilayer's structure type. The more interesting from this point of view is the angular dependence of the ellipsometric angle (Fig. 3b). These phase incursions are characterized by the refraction coefficient gradient, but determining the phase information in different polarizations one can define this local change of the refraction coefficient.

Using the geometry of scattering (see Fig. 1a), it should be noticed that optical wave has some uneven amplitude and phase distribution (it is a spherical wave of $\sin x / x$ type) and, consequently, in the area of interaction it has some finite set of scattering angles. Therefore, taking into account the effects of polarization axis rotation at the lens output:

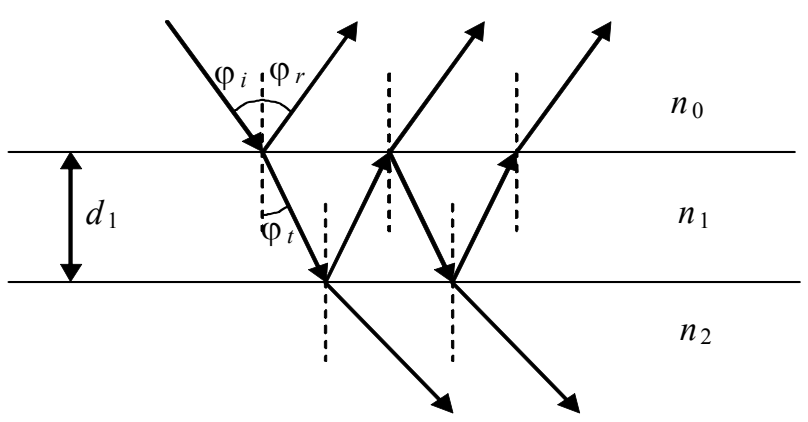

Fig. 2. Scheme of the light reflection from the multilayer structure.

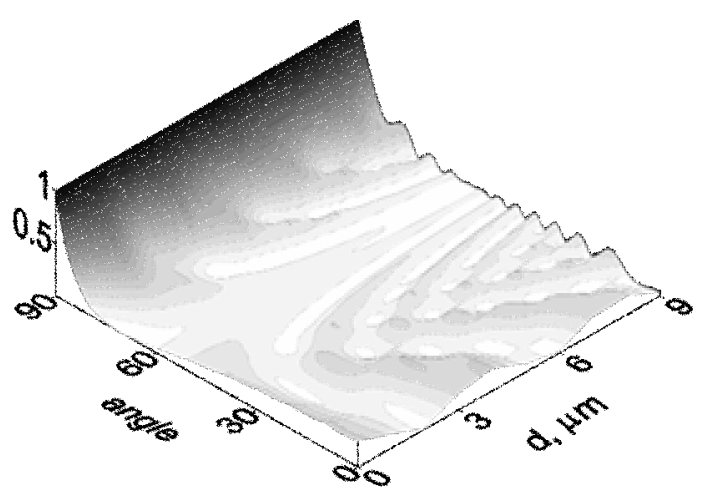

a)

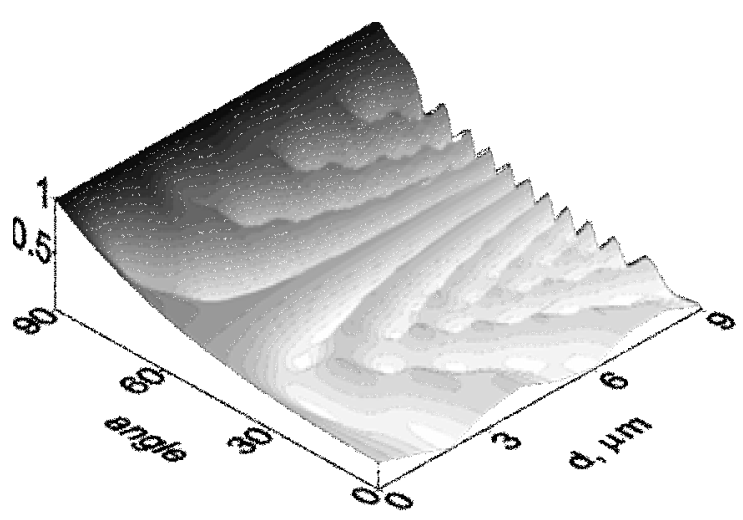

b)

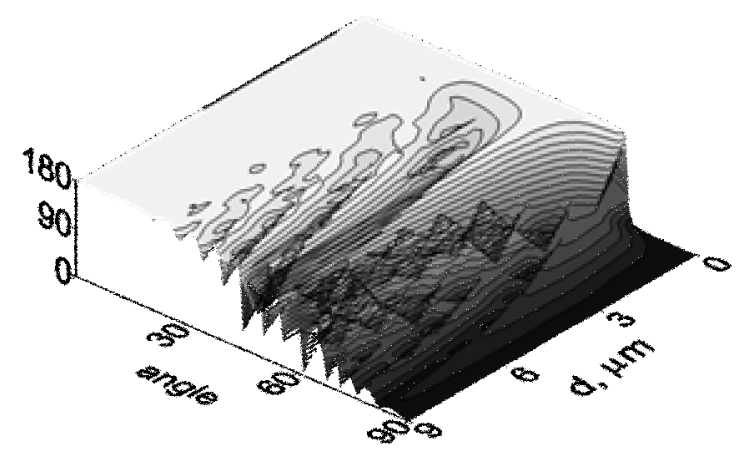

c)

Fig. 3. Dependences of orthogonal components for the reflection coefficients on the width of $\mathrm{SiO}_{2}$ layer(a) and ellipsometric angle $\Delta\left(\right.$ b) for the boundary air- $-\mathrm{SiO}_{2}-\mathrm{Si}$. 


$$
\begin{aligned}
& U_{\mathrm{II} x}(\vec{r})=\frac{1}{j \lambda r} \int U_{\mathrm{I} \xi}(\vec{\rho}) M_{\xi \xi}(\vec{\rho}) e^{-j \frac{2 \pi}{\lambda}|\vec{r}-\vec{\rho}|} \cos \left(\vec{k}_{\text {out }}, \vec{r}-\vec{\rho}\right) d^{2} \vec{\rho}, \\
& U_{\mathrm{II} y}(\vec{r})=\frac{1}{j \lambda r} \int U_{\mathrm{I \eta}}(\vec{\rho}) M_{\eta \eta}(\vec{\rho}) e^{-j \frac{2 \pi}{\lambda}|\vec{r}-\vec{\rho}|} \cos \left(\vec{k}_{\text {out }}, \vec{r}-\vec{\rho}\right) d^{2} \vec{\rho}, \\
& U_{\mathrm{II} z}(\vec{r})=\frac{1}{j \lambda r} \int\left(U_{\mathrm{I} \xi}(\vec{\rho}) M_{z \xi}(\vec{\rho})+U_{\mathrm{I \eta}}(\vec{\rho}) M_{z \eta}(\vec{\rho})\right) e^{-j \frac{2 \pi}{\lambda}|\vec{r}-\vec{\rho}|} \cos \left(\overrightarrow{k_{\text {out }}}, \vec{r}-\vec{\rho}\right) d^{2} \vec{\rho}
\end{aligned}
$$

can lead to the wave energy redistribution between the orthogonal components and though the scattered wave polarization change is eventual.

\section{Experimental setup and measurement principles}

The optical scheme of the experimental setup used for the surface investigations is shown in Fig. 4. The He-Ne laser $(\lambda=0.63 \mu \mathrm{m})$ beam intensity is adjusted by the polarization attenuator that consists of the $\mathrm{P} 1$ and $\mathrm{P} 2$ polarizers. After passing the $\lambda / 4$ plate, the laser beam becomes circularly polarized. P3 polarizer is used for extracting two orthogonal linearly polarized beams for illuminating the tested objects. After the circular aperture $\mathrm{D} 1$, the laser beam is divided into two beams. The wave reflected from the beam splitter BS1 is incident on the reference mirror $\mathrm{M}$, reflects from its surface and goes back to the system and forms the reference beam. The wave that passes through the beam splitter BS1 is focused on the surface of reflective object by the microscope objective L5. The system of L1 and L5 lenses and BS1BS2 beam splitters transposes the sample plane into the object plane of the microscope, which is used for visual control of the beam's focusing on the tested surface. The sample is fixed on the table with computer control and can be moved in two orthogonal directions in the plane normal to the beam axis. It is able to scan the surface with the step of about $10 \mu \mathrm{m}$ and several millimeters in aperture. The focused spot size can vary from 5 to $30 \mu \mathrm{m}$ depending on the D1 aperture size. The beam reflected from the sample after re-passing the microscope objective L5 forms the object wavefront that is transposed into the object plane of the scanner by the $4 \mathrm{~F}$ system of longfocus lenses L2-L3 and BS1 mirror. The scanner consists of the controlled diaphragm D2, the lenses L4 (focal length $200 \mathrm{~mm}$ ) and the CCD photodetector. The D2 diaphragm can be displaced in the plane normal to the beam and can be controlled by the computer. For the D2 diaphragm displacement the two-coordinate device with the scan step of $0.19 \mathrm{~mm}$ and $15 \times 15 \mathrm{~mm}$ aperture was used. The control of stepping motors was made via the parallel port of the personal computer (PC) by the developed controller and software.

\section{Results and discussion}

Testing with laser beams possessing linear orthogonal polarizations was made. The staggered structures on the photoresist layer with sizes $50 \times 50 \mu \mathrm{m}$ and spatial halfperiods of $l=0.8 \mu \mathrm{m}$ and $l=1.2 \mu \mathrm{m}$ were used (Fig. 5a)

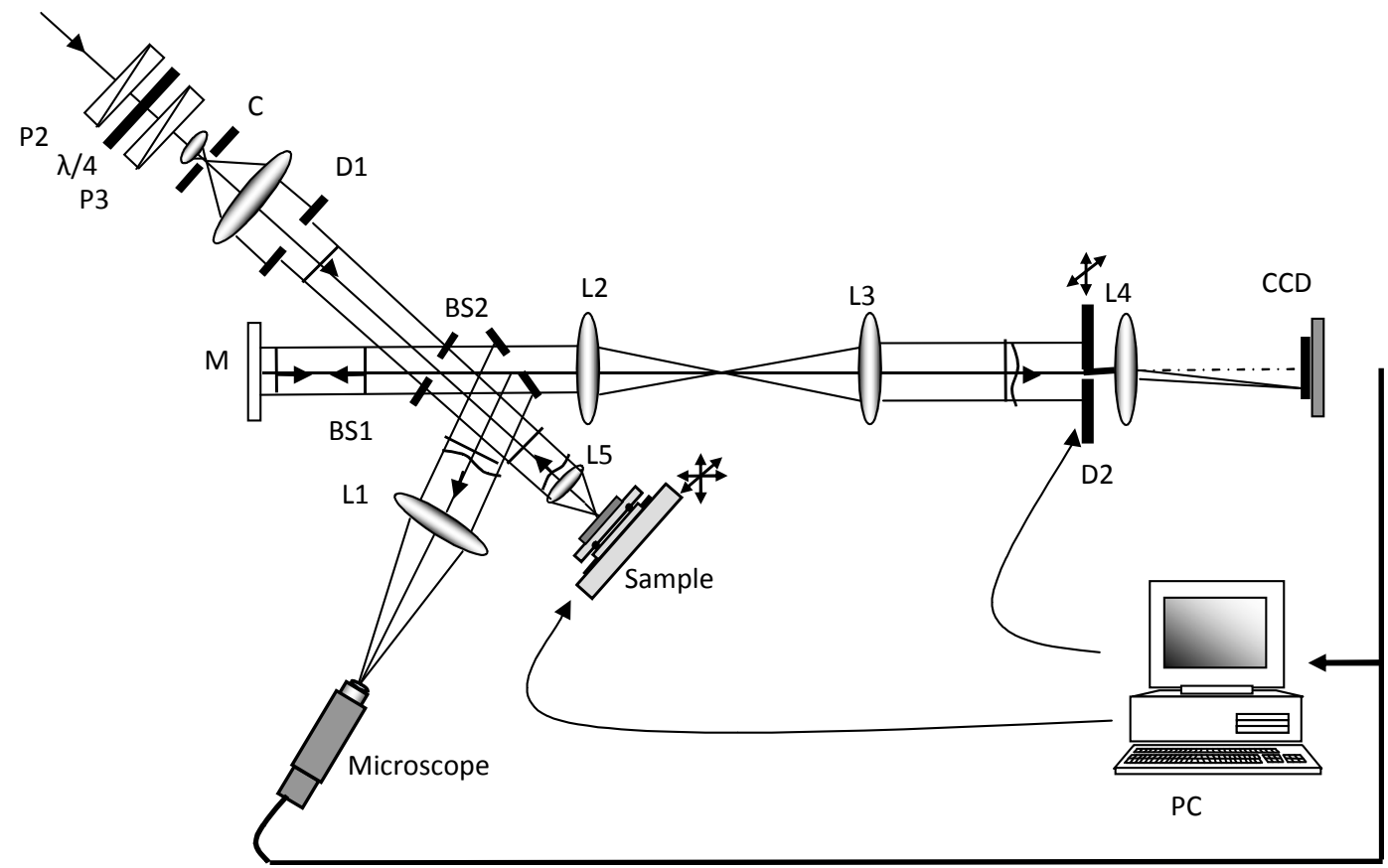

Fig. 4. Optical scheme of the experimental setup.

(C) 2010, V. Lashkaryov Institute of Semiconductor Physics, National Academy of Sciences of Ukraine 


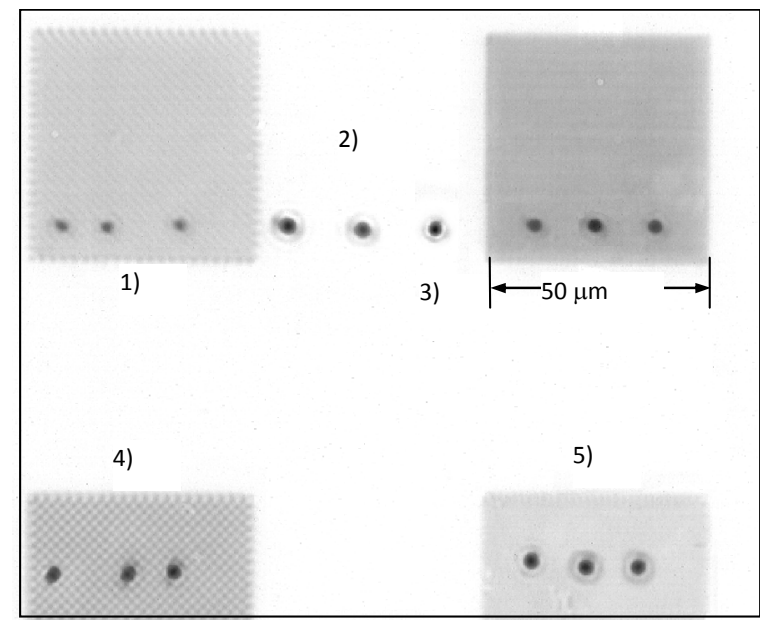

a)

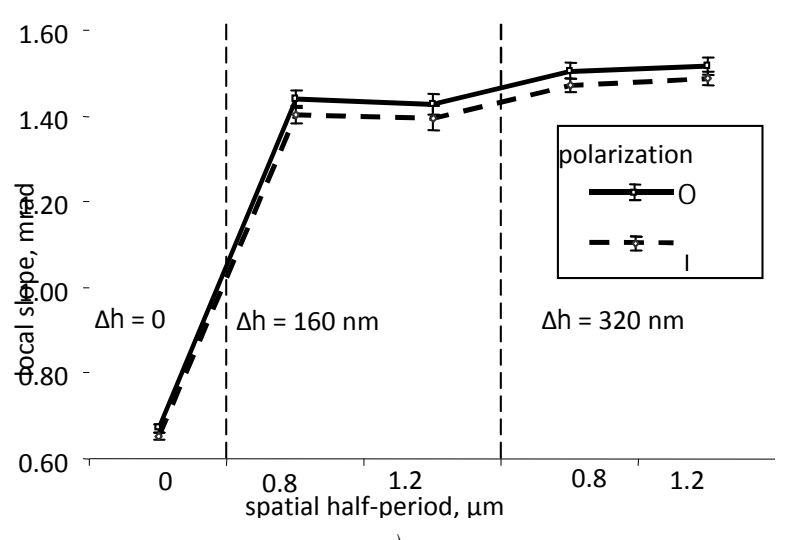

a)

Fig. 5. The surface area image (a): 1) $l=1.2 \mu \mathrm{m}, \Delta h=160 \mathrm{~nm}$; 2) clean surface; 3) $l=0.8 \mu \mathrm{m}, \Delta h=160 \mathrm{~nm}$; 4) $l=1.2 \mu \mathrm{m}$, $\Delta h=320 \mathrm{~nm}$; 5) $l=0.8 \mu \mathrm{m}, \Delta h=320 \mathrm{~nm}$; (b) values of the wavefront local slope for two orthogonal polarizations.

as a sample (relief depth $\Delta h$ was 160 and $320 \mathrm{~nm}$ ). The measurement was made in the following manner: the local slopes of the wavefront reflected from three spots on the clean surface and from three spots on each mentioned staggered structure were analyzed. And for each spot, a set of 5 wavefront realizations was recorded for considering the statistical data spread. Measurements were made in a certain wavefront subaperture on the distance of $1 \mathrm{~mm}$ from the optical axis, the scanner diaphragm diameter was $0.5 \mathrm{~mm}$.

Fig. $6 \mathrm{~b}$ shows the wavefront local slopes in the mentioned subaperture for two orthogonal polarizations of the probing beam on different structures of the sample with different half-period and relief depth. As one can see, the values of wavefront local slopes for the clean surface differ from the values for the structured surface. It also should be mentioned that wavefront local slope values are equal for two orthogonal linearly polarized components. This fact confirms that local slopes shift is caused by surface relief only.

\section{Conclusions}

The possibility of changes in the polarization state and full depolarization of the laser beam reflected from inhomogeneity with a changed refraction index is theoretically shown. The investigation of tested objects has shown the wavefront scanner ability to determine surface inhomogeneities, the structure of which is less that the focus spot size and has the appearance of stripes with different widths. The proposed wavefront scanner allows determining the surface features by the form of the wave reflected from its surface in two orthogonal polarizations. The obtained values of wavefront local slopes reflected from the tested sample are equal for two orthogonal polarizations; this fact confirms the "geometric" origin of present defects.

\section{References}

1. D.M. Alloin, J.-M. Mariotti (Eds.), Adaptive Optics for Astronomy. Kluwer Academic Publ., Dordrecht-Boston-London, 1994.

2. L. Thibos, Principles of Hartmann-Shack aberrometry // J. Refract. Surgery 16(5), p. 563-565 (2000).

3. C. Curatu, G. Curatu, J. Rolland, Fundamental and specific steps in Shack-Hartmann wavefront sensor design // Proc. SPIE 6288, No. 628801 (2006).

4. M.M. Kotov, V.N. Kurashov, D.V. Podanchuk, et al., Surface defective structure detection with Shack-Hartmann wavefront sensor // Bulletin of the University of Kiev. Series: Physics \& Mathematics No. 3, p. 341-348 (2006).

5. A.A. Goloborodko, V.I. Grygoruk, M.M. Kotov et al., Determination of local surface defects using a Shack-Hartmann wavefront sensor // Ukrainian Journal of Physics 53(10), p. 946-951 (2008).

6. M.M. Kotov, D.V. Podanchuk, N.S. Sutyagina et al., Wavefront scanner for testing the surface of optical elements // Proc. VIII Intern. Young Scientists' Conference on Applied Physics, Kyiv, p. 192 (2008).

7. V. Laude, S. Oliver, C. Dirson et al., Hartmann wave-front scanner // Opt. Lett. 24(24), p. 17961798 (1999).

8. T.D. Raymond, D.R. Neal, D.M. Topa, T.L. Schmitz, High-speed, non-interferometric nanotopographic characterization of $\mathrm{Si}$ wafer surfaces, Nanoscale Optics and Applications, Guozhong Cao, Wiley P. Kirk (Eds) // Proc. SPIE 4809 (2002).

9. N.S. Averkiyev, S.O. Kognovitskyi, V.V. Travnikov, Polarization anisotropy of optical reflection spectra for structures with open nano-fibers // Fizika i tekhnika poluprovodnikov 33(9), p. 10801083 (1999), in Russian.

10. J.W. Goodman, Statistical Optics. John Wiley and Sons, Inc., New York-Chichester-WeinheimBrisbane-Singapore-Toronto, 2000. 